\title{
Evidence for criticality in financial data
}

G. Ruiz ${ }^{1,2, a}$ and A.F. de $\operatorname{Marcos}^{3}$

${ }^{1}$ Departamento de Matemática Aplicada a la Ingeniería Técnica Aeronáutica, Universidad Politécnica de Madrid, Pza. Cardenal Cisneros 3, 28040 Madrid, Spain

${ }^{2}$ Centro Brasileiro de Pesquisas Fisicas, Rua Xavier Sigaud 150, Rio de Janeiro 22290-180, Brazil

${ }^{3}$ Escuela Técnica Superior de Ingeniería Aeronáutical y del Espacio, Universidad Politécnica de Madrid, Madrid, Spain

Received 21 February 2017 / Received in final form 20 September 2017

Published online 15 January 2018 - C EDP Sciences, Società Italiana di Fisica, Springer-Verlag 2018

\begin{abstract}
We provide evidence that cumulative distributions of absolute normalized returns for the 100 American companies with the highest market capitalization, uncover a critical behavior for different time scales $\Delta t$. Such cumulative distributions, in accordance with a variety of complex - and financial - systems, can be modeled by the cumulative distribution functions of $q$-Gaussians, the distribution function that, in the context of nonextensive statistical mechanics, maximizes a non-Boltzmannian entropy. These $q$ Gaussians are characterized by two parameters, namely $(q, \beta)$, that are uniquely defined by $\Delta t$. From these dependencies, we find a monotonic relationship between $q$ and $\beta$, which can be seen as evidence of criticality. We numerically determine the various exponents which characterize this criticality.
\end{abstract}

\section{Introduction}

The analysis of financial data by methods developed for physical systems has extensively attracted the interest of physicists [1-12]. One of the reasons for such interest is that statistical mechanics offers a productive insight to macroeconomic systems, as the particles from thermodynamic systems can be considered an analogue for macroeconomic systems such as companies, people, and states. A clear example of this approach is the analysis of the applicability of Fermi-Dirac distribution to income and wealth [13], which states that money distribution in an economy behaves similarly with the distribution of electrons in quantum systems. In this line, income and wealth distributions were extensively studied by Yakovenko [14-17] and Kusmartsev $[18,19]$ in terms of the Maxwell-Boltzmann, Bose-Einstein, and lognormal distributions. Properties of cumulative distributions of financial data have been also discussed, with the argument that power law tails should be always related to a critical behavior. A literature review is available in [20], with a theoretical framework of statistical mechanics distributions applied to income, wealth, and expenditure distribution.

In fact, financial markets are strongly fluctuating complex systems whose dynamics are difficult to understand because of the complexity of their internal elements and correlations, and also because of the many intractable external factors acting on them.

However, remarkably enough, the interactions between these various ingredients generate many observable whose

\footnotetext{
${ }^{a}$ e-mail: guiomar.ruiz@upm.es
}

statistical properties appear to be similar for quite different markets. Consequently, we are allowed to refer to some "universal" trends, on which we focus herein.

As a matter of fact, it has been observed, in financial data, that rare events give raise to pronounced tails in the appropriate probability distributions - tails that are in fact frequently found in complex systems. Such is the case of the return distributions associated with time series $[5,21]$ on varying time scales, $\Delta t$. These fat tails reveal long-range correlations that frequently cause standard statistical mechanics to be inadequate for describing them. This kind of scenario also emerges in the systems that permanently reside in the neighborhood of their critical point, where physical quantities present power-law dependencies of the type $f(x) \sim x^{-\tau}$, characterized by a critical exponent $\tau$.

In the present work, we will be concerned with the statistical analysis of the return distributions of the 100 most capitalized companies traded on NYSE in 1998-1999. ${ }^{1}$ Our objective is to uncover some empirical laws that seem to govern such financial markets.

\footnotetext{
${ }^{1}$ They were not taken from any specific index, their capitalization being the sole criterion. Their 1999 tickers are as follows: AA, ABT, AHP, AIG, ALD, AMGN, AOL, ARC, AT, AUD, AXP, BA, BAC, BEL, BK, BLS, BMY, BUD, C, CA, CBS, CCL, CCU, CHV, CL, CMB, CMCS, COX, CPQ, CSCO, DD, DELL, DH, DIS, DOW, EDS, EK, EMC, EMR, ENE, F, FBF, FNM, FRE, FTU, G, GCI, GE, GLW, GM, GTE, GTW, HD, HWP, IBM, INTC, JNJ, JPM, KMB, KO, LLY, LOW, LU, MCD, MDT, MER, MMC, MMM, MO, MOT, MRK, MSFT, MTC, MWD, ONE, ORCL, PEP, PFE, PG, PNU, QCOM, QWST, SBC, SCH, SGP, SLB, SUNW, T, TWX, TX, TXN, UMG, USW, UTX, VIAB, WCOM, WFC, WMT, XON, YHOO. Their returns were calculated according to the standard procedure, from stock prices sampled with a given frequency.
} 
Following along the lines of reference [22], we will apply the so called nonextensive statistical mechanical analysis $[23,24]$ to the empirical distribution data of the mentioned normalized returns. The characteristic generalized distribution functions of nonextensive statistics ubiquitously emerge in complex systems, and very especially as economical and financial stylized features, including price returns and volume distributions, interoccurrence times, characterization of wealth distributions and associated inequalities among others $[25,26]$. Remarkably, time series of prizes, conveniently replaced by their corresponding returns, have in fact demonstrated to be robustly explained by the nonextensive statistics [22,26-28].

The paper is organized as follows. In Section 2 we briefly review the essential concepts and tools of nonextensive statistics to be used. In Section 3 we show the procedure to calculate the generalized cumulative and non cumulative distributions of the returns of the referred financial market, and their criticality due to the fact that - in analogy to critical phenomena - for each value of the sampling time there exists just one value of the generalized temperature. Our main conclusions are drawn in Section 4.

\section{Nonextensive statistics and complex systems}

Nonextensive statistical mechanics [23,24], a current generalization of the Boltzmann-Gibbs (BG) statistical mechanics when its associated entropy $S_{B G}$ does not obey the standard asymptotic behavior $S_{B G}(N) \sim N$ for $N \rightarrow \infty$ ( $N$ being the number of elements), occurs to be useful in the description of complex financial systems $[27,29-33]$.

The theory has been developed around the concept of nonadditive entropy, which is maximized, with the appropriate constraints [34], by the family of the $q$-Gaussian distributions

$$
G_{q}(x)=A\left(q, \beta_{q}\right) \exp _{q}\left[-\beta_{q}\left(x-\mu_{q}\right)^{2}\right],
$$

where $q$ is a characteristic index, $\beta_{q}$ is a sort of inverse temperature [35], $\mu_{q}$ is the escort averaged first moment $[36], A(q, \beta)$ is a normalization factor, and the function

$$
\exp _{q}(x) \equiv[1+(1-q) x]_{+}^{1 /(1-q)}
$$

with $[x]_{+}=x$ if $x>0$ and $[x]_{+}=0$ otherwise, is a generalization of the exponential function - the $q \rightarrow 1$ limit makes $\exp _{q}(x) \rightarrow \exp (x)$. The normalization factor in equation (1) reads [37], for the values of $q$ we are now involved $(1<q<3)$ :

$$
A\left(q, \beta_{q}\right)=\sqrt{\frac{q-1}{\pi} \beta_{q}} \frac{\Gamma\left[\frac{1}{q-1}\right]}{\Gamma\left[\frac{3-q}{2(q-1)}\right]} .
$$

The $q$-Gaussian distribution (1) generalizes the Gaussian distribution in a similar way as nonadditive entropy generalizes $S_{B G}[23]$.
In fact, the $q \rightarrow 1$ limit makes equations (1)-(3) to recover the Gaussian distribution, i.e. $G_{1}(x)=$ $1 /\left(\sigma_{1} \sqrt{2 \pi}\right) \exp \left[\left(x-\mu_{1}\right)^{2} /\left(2 \sigma_{1}^{2}\right)\right]$. A generalization of the Central Limit Theorem, where the $q$-Gaussian distributions themselves become their attractors, has already been formulated [38-40].

\section{Criticality and absolute normalized returns}

The absolute normalized returns are conventionally defined in the following manner. For the time series $W(t)$ that represent the prizes - or the market index value - at time $t$, the returns over a sample interval $\Delta t, R_{\Delta t}(t)$, are defined as

$$
\begin{aligned}
R_{\Delta t}(t) & \equiv \ln W(t+\Delta t)-\ln W(t) \\
& \simeq \frac{W(t+\Delta t)-W(t)}{W(t)}
\end{aligned}
$$

where the approximation holds for small variations of $W(t)$. By centering and normalizing $R_{\Delta t}(t)$, to have unit variance, the normalized returns are obtained:

$$
r_{\Delta t}(t) \equiv\left[R_{\Delta t}(t)-\left\langle R_{\Delta t}(t)\right\rangle_{T}\right] / v_{\Delta t},
$$

where $\langle\cdots\rangle_{T}$ denotes a time average, and volatility $v_{\Delta t}$ is the standard deviation of the returns over the period $T$.

In the spirit of Figure 61 in reference [22], we are interested in studying the cumulative distribution functions (CDF) of the absolute normalized returns, for different time scales $\Delta t$, of the 100 American companies with the highest market capitalization. In other words, we analyze the probability for an absolute return to be larger than a threshold $x$, i.e. $C D F(x) \equiv P\left(\left|r_{\Delta t}\right|>x\right)$. The negative and positive wings of empirical distributions are supposed to present negligible quantitative discrepancies and, consequently, we focus on the analysis of absolute returns.

The asymptotic behavior of such normalized returns has been observed to follow an asymptotical power-law-like dependence of the type $C D F(x) \sim 1 / x^{\alpha}(\alpha>0)$. This is but one of the arguments that make $q$-Gaussian distributions attractive to describe them; indeed $q$-Gaussians asymptotically develop a power-law form $G_{q}(x) \sim x^{2 / 1-q}$ for $(x \gg 1)$.

First, we analytically obtain the CDF of a $q$-Gaussian probability distribution function (pdf), with $\mu=0$ and re-normalized temperature $\beta^{-1}$, as

$$
P\left(\left|r_{\Delta t}\right|>x\right)=1-2 A(q, \beta) x_{2} F_{1}(\alpha, \delta ; \gamma, \tau),
$$

where $\alpha=1 / 2, \delta=1 /(q-1), \gamma=3 / 2, \tau=\beta(1-q) x^{2}$ and where ${ }_{2} F_{1}(\alpha, \delta ; \gamma, \tau)$ is the hypergeometric function. Equation (6) provides a $q$-dependent asymptotical $(x \gg 1)$ behavior of the type $\sim x^{(q-3) /(q-1)}$, that fits the $\alpha$-dependent asymptotical behavior of absolute normalized returns, and provides the $q$-Gaussian index through 
Table 1. Time scales of absolute normalized returns, and the $(q, \beta)$ values of their respective estimated CDFs. The $q$ index is obtained by a least square fitting of the tail distribution. The $\beta$ index is obtained by fitting the whole CDF.

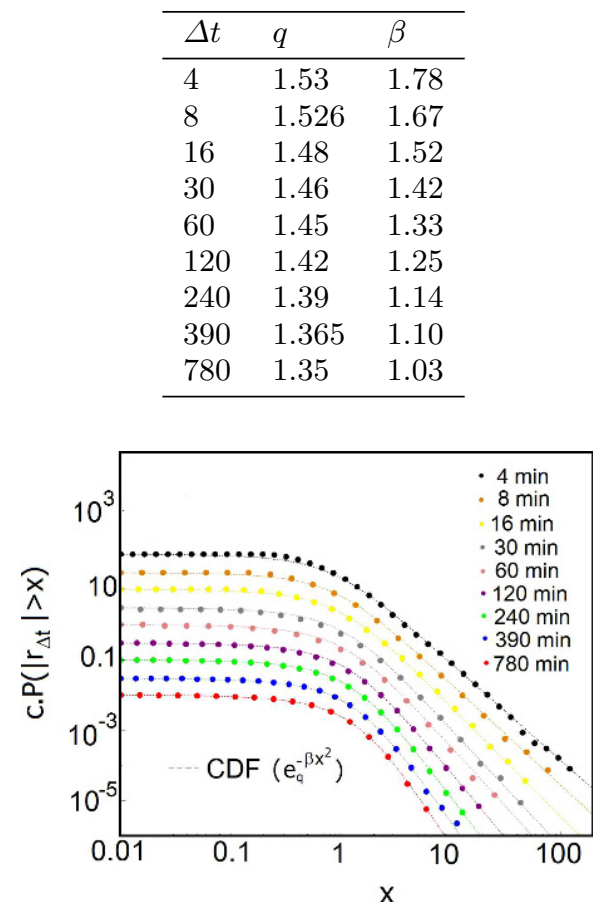

Fig. 1. Cumulative distributions of absolute normalized returns that correspond to different time scales $\Delta t$ for the 100 American companies with the highest market capitalization (points), and the fitted cumulative $q$-Gaussian distributions (lines). In order to better visualize the results, each $q$-Gaussian $\mathrm{CDF}$ and the respective experimental data have been multiplied by a positive factor, $c \neq 1$.

the relation:

$$
q=\frac{3+\alpha}{1+\alpha}
$$

Even in the case that empirical data of a particular time scale did not attain the asymptotical behavior yet, we observe that cumulative distributions are also properly fitted by the CDF of a $q$-Gaussian pdf (6). We obtain the value of $\beta$ associated to the index $q$ that corresponds to each time scale $\Delta t$, by a least squares fitting technique. Our $q$ versus $\Delta t$ results (see Tab. 1 ), are in a quite satisfactory agreement with reference [22].

The fitted CDFs are represented, for all time scales, in Figure 1, together with the experimental data provided in reference [22]. The convergence of the CDF exhibits that, as $\Delta t$ increases, the value of $q$ decreases. Hypothetical final convergence to a Gaussian $(q \rightarrow 1)$ appears to be abrupt (see Fig. 2).

Figures 3 and 4 exhibit the good agreement with the $q$-Gaussian pdfs that lead to equation (6), with respect to the derivatives of the experimental cumulative distributions.

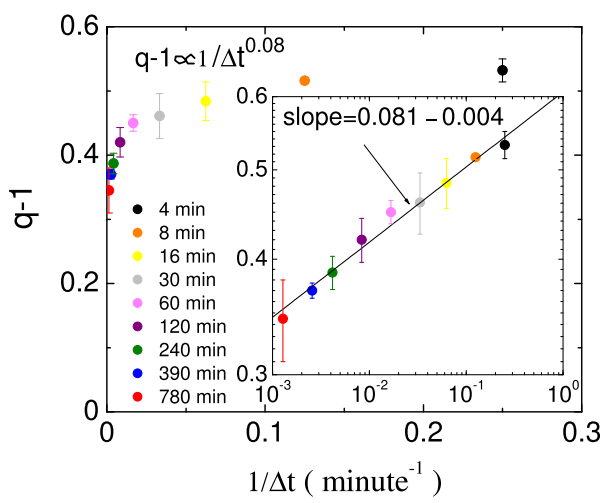

Fig. 2. Dependence, versus temporal scale $\Delta t$, of nonextensive index $q$, for the estimated $q$-Gaussian pdfs of normalized absolute returns. Inset: $\log -\log$ representation shows a power-law dependence of the type $q-1 \propto \Delta t^{-\tau}$, with $\tau=0.081 \pm 0.004$.

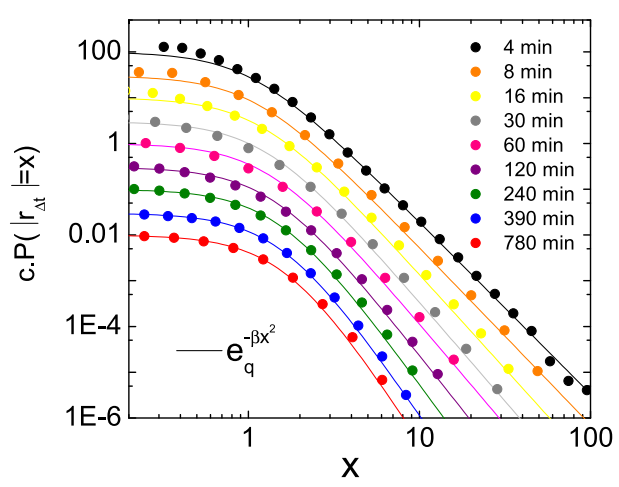

Fig. 3. Log- $\log$ representation of the probability density distributions of absolute normalized returns for different time scales $\Delta t$. Points have been obtained by numerical derivation of cumulative values. Lines represent the $q$-Gaussian pdfs that lead to the fitted CDFs in Figure 1. In order to better visualize the results, each $q$-Gaussian and the respective numerically estimated values, are multiplied by a positive factor $c \neq 1$.

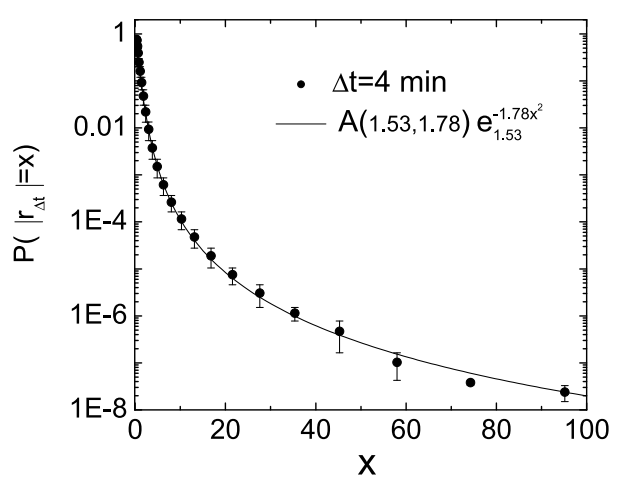

Fig. 4. Log-linear representation of the probability density distribution of absolute normalized returns, for time scale $\Delta t=4$. Points have been obtained by numerical derivation of cumulative values. Line represents the $q$-Gaussian pdf that leads to the corresponding CDF in Figure 1, i.e. $q=1.53$ and $\beta=1.78$. The error bars are generated by the diversity of numerical methods to obtain the derivatives (points) of the cumulative distribution values (forward, backward and central differences). 


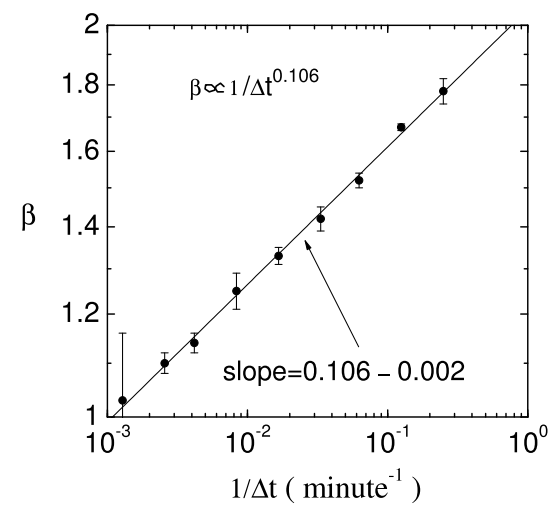

Fig. 5. Log-log representation of the re-normalized inverse temperature $\beta$ versus temporal scale $\Delta t$, for the estimated $q$ Gaussian pdfs of normalized absolute returns. A power-law dependence of the type $\beta^{-1} \propto \Delta t^{-\gamma}$ is observed, with $\gamma=$ $0.106 \pm 0.002$.

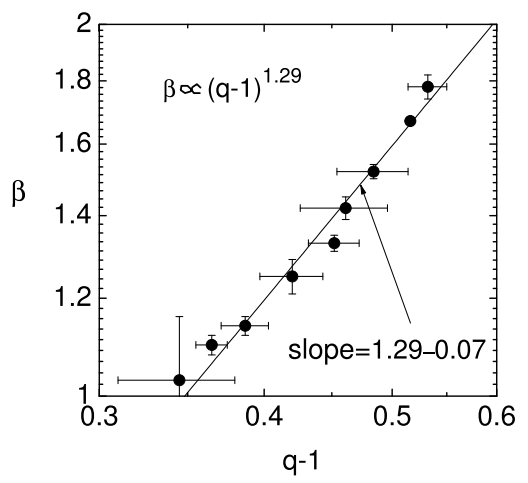

Fig. 6. Log- $\log$ representation of the re-normalized inverse temperature $\beta$ versus $q-1$, for the estimated $q$-Gaussian pdfs of normalized absolute returns. A power-law dependence of the type $\beta^{-1} \propto(q-1)^{-\delta}$ is observed, with $\delta=1.29 \pm 0.07$.

We have also observed that simple relations exist between the quantities $(q, \beta)$ involved for each time scale $\Delta t$. A power-law dependence is observed for both $q$ and $\beta$ as a function of time scale $\Delta t$, the exponents being $\tau=0.081 \pm 0.004$ (Fig. 2) and $\gamma=0.106 \pm 0.002$ (Fig. 5). Figure 6 shows in fact that the re-normalized temperature $\beta^{-1}$ is not a free value, but it also exhibits a powerlaw dependence versus $q$, mainly $\beta^{-1}=(q-1)^{-\delta}$, with $\delta=1.29 \pm 0.07$.

\section{Conclusions}

Our results show that $q$-statistics describes complex behaviour that emerges in the analysis of the present particular financial data. Similar results have been previously obtained in references $[22,27,30]$. But, undoubtedly, the novelty of the present results is that we have also exhibited that both parameters $(q, \beta)$ of the nonextensive scenario are specific values that are fixed by $\Delta t$. Such behavior is analogous to the behavior of a variety of other systems that are properly described by $q$-statistics, for example scale-free $d$-dimensional geographically-located networks [41], quark-gluon soup in high-energy particle collisions [42], LHC/CERN and RHIC/Brookhaven experiments [43] and anomalous diffusion in confined granular media [44]. Another simple and paradigmatic example is the logistic map where, as a reminiscence of this type of behavior, the $q$-generalized Lyapunov exponent depends on the value of $q$ that characterizes the sensitivity to initial conditions at the edge of chaos $[45,46]$. This frequent feature comes from the fact that $q$-statistics typically emerges at critical-like regimes and appears to be deeply related to an hierarchical occupation of phase space.

We are grateful to professors J. Kwapień and S. Drożdż for sharing with us their empirical cumulative distribution data. One of us (G.R.) is grateful to professor C. Tsallis for is fruitful suggestions. One of us (G.R.) also acknowledges the warm hospitality at the CBPF (Brazil) and the partial financial support by the John Templeton Foundation (USA).

\section{Author contribution statement}

All the authors were involved in the preparation of the manuscript. All the authors have read and approved the final manuscript.

\section{References}

1. R.N. Mantegna, H.E. Stanley, An introduction to econophysics: correlations and complexity in finance (Cambridge University Press, Cambridge, 2000)

2. H. Takayasu, Empirical science of financial fluctuations: the advent of econophysics (Springer, Berlin, 2002)

3. A. Bunde, H.J. Schellnhuber, J. Kropp, The science of disasters: climate disruptions, hearth attacks and market crashes (Springer, Berlin, 2002)

4. L. Bachelier, Ann. Sci. École Norm. Suppl. 3, 21 (1900)

5. B.B. Mandelbrot, J. Bus. 36, 294 (1962)

6. A. Pagan, J. Empir. Finance 3, 15 (1996)

7. X. Gabaix, P. Gopikrishnan, V. Plerou, H.E. Stanley, Nature 423, 267 (2003)

8. P. Gopikrishnan, M. Meyer, L.A.N. Amaral, H.E. Stanley, Eur. Phys. J. B 3, 138 (1998)

9. P. Gopikrishnan, V. Plerou, L.A.N. Amaral, M. Meyer, H.E. Stanley, Phys. Rev. E 60, 5305 (1999)

10. M. Denys, T. Gubiec, M. Jagielski, R. Kutner, H.E. Stanley, Phys. Rev. E 94, 042305 (2016)

11. P. Oświecimka, J. Kwapień, S. Drożoż, Physica A 347, 626 (2005)

12. J. Kwapień, P. Oświecimka, S. Drożoż, Physica A 350, 466 (2005)

13. E. Oltean, F.V. Kusmartsev, H.I.J.E.N.E. 5, 221 (2012)

14. A. Dragulesku, V.M. Yakovenko, Statistical mechanics of money, income, and wealth: a short survey, http://www2.physics.umd.edu/ yakovenk/econophysics/

15. A. Dragulescu, V.M. Yakovenko, Eur. Phys. J. B 17, 723 (2000)

16. A. Dragulescu, V.M. Yakovenko, Eur. Phys. J. B 20, 585 (2001)

17. A.C. Silva, V.M. Yakovenko, Europhys. Lett. 69, 304 (2005) 
18. K.E. Kurten, F.V. Kutmartsev, Europhys. Lett. 93, 28003 (2011)

19. F.V. Kutmartsev, Phys. Lett. A 375, 966 (2011)

20. E. Oltean, Modelling income, wealth, and expenditure data by use of econophysics arXiv: 1603.08383 [q-fin.GN] (2016)

21. B.B. Mandelbrot, Fractals and scaling in finance (Springer, Berlin, 1997)

22. J. Kwapień, S. Drożdż, Phys. Rep. 515, 115 (2012)

23. C. Tsallis, J. Stat. Phys. 52, 479 (1988)

24. C. Tsallis, Introduction to nonextensive statistical mechanics - approaching a complex world (Springer, New York, 2009)

25. C. Tsallis, Entropy 19, 457 (2017)

26. S.M. Duarte Queiros, Quant. Finance 5, 475 (2005)

27. C. Tsallis, C. Anteneodo, L. Borland, R. Osorio, Physica A 324, 89 (2003)

28. R. Osorio, L. Borland, C. Tsallis, Distributions of height-frequency stock-market observables, in Nonextensive entropy - interdisciplinary applications, edited by M. Gell-Mann, C. Tsallis (Oxford University Press, New York, 2004)

29. F. Michael, M.D. Johnson, Physica A 320, 525 (2003)

30. R. Rak, S. Drożdż, J. Kwapień, Physica A 374, 315 (2007)

31. S. Drożdż, M. Forczek, J. Kwapień, P. Oświecimka, R. Rak, Physica A 383, 59 (2007)
32. J. Ludescher, C. Tsallis, A. Bunde, Europhys. Lett. 95, $68002(2011)$

33. C. Tsallis, Chaos Solitons Fractals 88, 254 (2016)

34. C. Tsallis, S.V.F. Levy, A.M.C. Souza, R. Maynard, Phys. Rev. Lett. 75, 3589 (1995)

35. C. Tsallis, R.S. Mendes, A.R. Plastino, Physica A 261, $534(1998)$

36. C. Tsallis, A.R. Plastino, R.F. Alvarez-Estrada, J. Math. Phys. 50, 043303 (2009)

37. D. Prato, C. Tsallis, Phys. Rev. E 60, 2398 (1999)

38. L.G. Moyano, C. Tsallis, M. Gell-Mann, Europhys. Lett. 73, $813(2006)$

39. S. Umarov, C. Tsallis, S. Steinberg, Milan J. Math. 76, 307 (2008)

40. S. Umarov, C. Tsallis, J. Phys. A: Math. Theor. 49, 415204 (2016)

41. S.G.A. Brito, L.R. da Silva, C. Tsallis, Nat. Sci. Rep. 6, $27992(2016)$

42. D.B. Walton, J. Rafelshi, Phys. Rev. Lett. 84, 31 (2000)

43. V. Khachatryan et al. (CMS Collaboration), Phys. Rev. Lett. 105, 022002 (2010)

44. G. Combe, V. Richefeu, M. Stasiak, A.P.F. Atman, Phys. Rev. Lett. 115, 238301 (2016)

45. M.L. Lyra, C. Tsallis, Phys. Rev. Lett. 80, 53 (1998)

46. F. Baldovin, A. Robledo, Phys. Rev. E 69, 045202(R) (2004) 